\title{
The CCND1 c.870G risk allele is enriched in individuals of African ancestry with plasma cell dyscrasias
}

\author{
Linda B. Baughn', Zhuo Li ${ }^{2}$, Kathryn Pearce ${ }^{1}$, Celine M. Vachon ${ }^{3}$, Mei-Yin Polley ${ }^{3}$, Jonathan Keats $\mathbb{1 0}^{4}$, Eran Elhaik, \\ Michael Baird ${ }^{6}$, Terry Therneau ${ }^{3}$, James R. Cerhan $\mathbb{1}^{3}$, P. Leif Bergsagel $\mathbb{1}^{7}$, Angela Dispenzieri ${ }^{8}$, S. Vincent Rajkumar (10) \\ Yan W. Asmann² and Shaji Kumar $\mathbb{0}^{8}$
}

\section{Dear Editor,}

Plasma cell (PC) dyscrasias, including multiple myeloma (MM), represent a spectrum of monoclonal gammopathies resulting from a clonal expansion of an abnormal plasma cell clone ${ }^{1}$. MM is generally preceded by precursor conditions known as monoclonal gammopathy of undetermined significance (MGUS) and smoldering MM (SMM) and is the most common hematologic malignancy in African Americans (AAs) $)^{2,3}$. It has been established that AAs, including Ghanaian men, display a 2-3-fold higher prevalence of MGUS with a reported similar risk of MGUS to MM progression compared to European Americans $(E A s)^{4-6}$. An increased MM incidence has also been observed in individuals with a family history of MM, an effect that was greater for individuals who self-identify as black ${ }^{7}$, suggesting that constitutional, MM susceptibility risk allele/s may explain the increased prevalence of MGUS among individuals of African ancestry. Interestingly, AAs also appear to have either a similar or in some cases superior survival outcome compared to EAs depending on patient age, specific treatment modalities and access to care including clinical trial enrollment ${ }^{3,8-10}$. This disparity in outcome may be explained by genetic differences that predispose to specific acquired, cytogenetically defined subtypes, which can influence disease prognosis and response to treatment. These cytogenetic subtypes can be broadly classified into either hyperdiploid

\footnotetext{
Correspondence: Shaji Kumar (Kumar.Shaji@Mayo.edu)

'Division of Laboratory Genetics, Department of Laboratory Medicine and Pathology, Mayo Clinic, Rochester, MN, USA

${ }^{2}$ Division of Biomedical Statistics and Informatics, Department of Health Sciences Research, Mayo Clinic, Jacksonville, FL, USA
}

Full list of author information is available at the end of the article. (characterized by gains of odd-numbered chromosomes) or translocations involving the immunoglobulin heavy chain $(I g H)$ gene on chromosome 14. In the context of $\mathrm{MM}$, hyperdiploidy and translocations $\mathrm{t}(11 ; 14)(\mathrm{q} 13 ; \mathrm{q} 32)$ and $\mathrm{t}(6 ; 14)(\mathrm{p} 21 ; \mathrm{q} 32)$ are typically associated with a favorable prognosis, while translocations $\mathrm{t}(4 ; 14)(\mathrm{p} 16 ; \mathrm{q} 32)$, $\mathrm{t}(14 ; 16)(\mathrm{q} 32 ; \mathrm{q} 23)$, or $\mathrm{t}(14 ; 20)(\mathrm{q} 32 ; \mathrm{q} 12)$ translocations are generally associated with an unfavorable prognosis ${ }^{1}$.

Many previous studies evaluating the mechanism of racial disparities in MGUS/MM have assessed race using self-reported demographic data rather than utilizing ancestry informative single-nucleotide polymorphisms (SNPs) as a method to determine the proportion of racial admixture. Using SNP data, we have recently identified a higher prevalence of $I g H$ translocations $\mathrm{t}(11 ; 14), \mathrm{t}(14 ; 16)$ and $t(14 ; 20)$ in individuals with $\geq 80 \%$ African ancestry ${ }^{11}$. Enrichment of $\mathrm{t}(11 ; 14)$ was confirmed in a more recent study $^{12}$. As an extension to our previous work ${ }^{11}$, we investigated which, if any, of the germline risk alleles previously associated with MM or MGUS risk, survival or risk of development of $\mathrm{t}(11 ; 14)^{13}$ (Supplemental Table 1) are enriched in individuals of African ancestry in our cohort of patients with plasma cell dyscrasias.

Genotype and ancestry information were obtained from the Precision Medicine Research Array (PMRA) data of 898 samples from patients with an abnormal plasma cell proliferative disorder fluorescence in situ hybridization (FISH) result and a concurrent conventional G-banded chromosome study (Supplemental materials and methods). Eight-hundred eighty-one of these samples were previously described ${ }^{11}$. As expected, the patient demographics of the full 898 sample cohort were similar to prior results ${ }^{11}$, including the increased 
risk of development of either a $t(11 ; 14), t(14 ; 16)$ or $t(14 ; 20)$ in individuals with high African ancestry $(\geq 80 \%$, very African cohort) compared to individuals with low African ancestry $(<0.1 \%,<30 \%$ Asian ancestry, European cohort) (Supplemental Tables 2 and 3).

Of the 18 previously reported SNPs examined within our cohort (Supplementary Table 1), 11 risk alleles were associated with European ancestry $(<0.1 \%$ African and $<30 \%$ Asian ancestries), involving variants at $2 \mathrm{p} 23.3,2 \mathrm{q} 12.3$, 3q26.2, 5q15, 6p21.33, 6q21, 10p12.1, 16q23.1, and 17p11.2 (Supplemental Table 4). Seven risk alleles were found associated with African ancestry ( $\geq 80 \%$ African ancestry) involving variants at 3p22.1, 3q26.2, 7p15.3, 8q24.21, 11q13.3, 16p13.11, and 17p11.2 (Supplemental Table 5). Of these seven variants, rs9344 at 11q13.3 encoding the CCND1 c.870G > A polymorphism was of particular interest due to the enrichment of the G-risk allele among persons of African ancestry (Table $1 \mathrm{~A}, p$-value $<0.0001$ ) and its association with increased risk of $t(11 ; 14)^{13}$.

Of the seven risk alleles associated with African ancestry, only rs 9344 was shown to be associated with $t(11 ; 14)$ in our full patient cohort with a G-risk allele frequency of 0.58 in non-t $(11 ; 14)$ controls compared to 0.73 in $\mathrm{t}(11 ; 14)$ cases (Table 1B, $p$-value $<0.0001$ ). The six additional risk alleles associated with African ancestry were not found to be associated with $t(11 ; 14)$ (Supplemental Table 6). The G allele correlated with $\mathrm{t}(11 ; 14)$ in both European $(p$-value $=$ 0.0048 ) and African ( $p$-value $=0.0522$ ) cohorts (Supplemental Table 7). A multivariate model including age, gender, race, and rs9344 identified rs9344 as significantly associated with $\mathrm{t}(11 ; 14)$ after adjusting for age, gender, and race group (Table 2) ( $p$-value $<0.001$ for GG and $p$-value $=$ 0.005 for AG genotypes).

To test if these results are replicable, we employed the Multiple Myeloma Research Foundation (MMRF) CoMMpass cohort, which includes individuals with newly diagnosed MM. Although the $t(11 ; 14)$ was not enriched in selfreported black individuals from this cohort (Supplemental Table 8) $(17.5 \%$ Black vs. $20.4 \%$ White, $p$-value $=0.47)$, there was a significant association between the SNP rs9344 and self-reported black race (Supplemental Tables 8 and 9) ( $p$-value $<0.0001)$ and with $\mathrm{t}(11 ; 14)$ when the $\mathrm{t}(11 ; 14)$ was analyzed as both total number of supporting read pairs $(p$-value $=0.0004)$ and presence or absense of $\mathrm{t}(11 ; 14)$ identified by long insert whole-genome sequencing (Supplemental Table 9) $(p$-value $=0.0001)$.

Here, we evaluated the prevalence of 18 previously published germline MGUS/MM risk alleles in our cohort of 898 patients stratified by genetically inferred ancestry rather than self-reported race data. Using this stratification approach, we confirm the association of the CCND1 c.870G risk allele (SNP rs9344) with cytogenetic subtype $t(11 ; 14)$ in both European and African populations. The frequency of the G-risk allele in our European cohort was similar to the reported risk allele frequency in the UK and German cohorts $^{13}$. Our study is the first to demonstrate that the G-risk allele is associated with African ancestry. This overall increased frequency of the G-risk allele in individuals with the highest African ancestry (0.81) vs. lowest African ancestry $(0.59)(p<0.0001)$ suggests that this risk allele may contribute to the increased prevalence of $t(11 ; 14)$ in AAs. Two other SNPs rs649392 and rs1352075 within CCND1 that are in linkage disequilibrium with rs9344 were also significantly correlated with $t(11 ; 14)$ and African ancestry (data not shown). No significant correlation was found between CCND1 SNPs rs9344, rs649392, or rs1352075 in association with $\mathrm{t}(14 ; 16)$ or $(14 ; 20)$ cytogenetic subtypes (data not shown).

Although we validate the association between rs9344 and self-reported black race and with $t(11 ; 14)$ in the CoMMpass study, we did not observe increased $t(11 ; 14)$ with self-reported black race. One possibility for this finding may be the presence of a smaller number of patients that self-reported as black with very high African ancestry. As reported by Manojlovic et al. ${ }^{9}$ there were $<20$ patients with $\geq 80 \%$ African ancestry in the CoMMpass IA9 dataset. Given that the CCND1 rs9344 polymorphism is a common allele and does not contribute to $t(11 ; 14)$ risk in a fully penetrant manner, it is probable that additional variants present in individuals with $\geq 80 \%$ African ancestry also contribute $\mathrm{t}(11 ; 14)$ risk.

The CCND1 gene encodes the cyclin D1 protein, a member of the cyclin D family, which regulate cyclindependent kinases (Cdks). Cyclin D in concert with Cdks phosphorylate the retinoblastoma protein promoting cell cycle progression ${ }^{14}$. MM tumors typically deregulate one of three cyclin D proteins ${ }^{15}$ and $t(11 ; 14)$ translocation is one mechanism promoting cyclin D1 deregulation ${ }^{16}$. The c.870G > A polymorphism is at chr11:69,462,910 (GRCh37/hg19) of the last nucleotide of exon 4 of CCND1 (NM_053056). Although both 870G and 870A encode the proline amino acid, the $\mathrm{G}$ allele has been reported to cause a novel splice donor site resulting in a longer cyclin D1a transcript ${ }^{14}$. The A allele has been reported to hinder splicing of exon 4 resulting in intron 4 read-through creating a truncated D1b transcript producing a protein shorter than D1a and lacking the D1a carboxy-terminus ${ }^{14}$. The lack of the carboxy-terminus of the D1b isoform results in nuclear retention ${ }^{17}$. Most studies have associated the A allele with cancer risk and poor outcome, with only a few studies correlating the $G$ allele to increased cancer risk ${ }^{14}$. Of interest, the $G$ allele has been associated with increased risk of cervical, head and neck and colorectal cancers, malignancies AAs have also been reported with increased incidence compared to $\mathrm{EAs}^{14}$.

To our knowledge, our study includes the largest group of individuals of African ancestry with an abnormal 


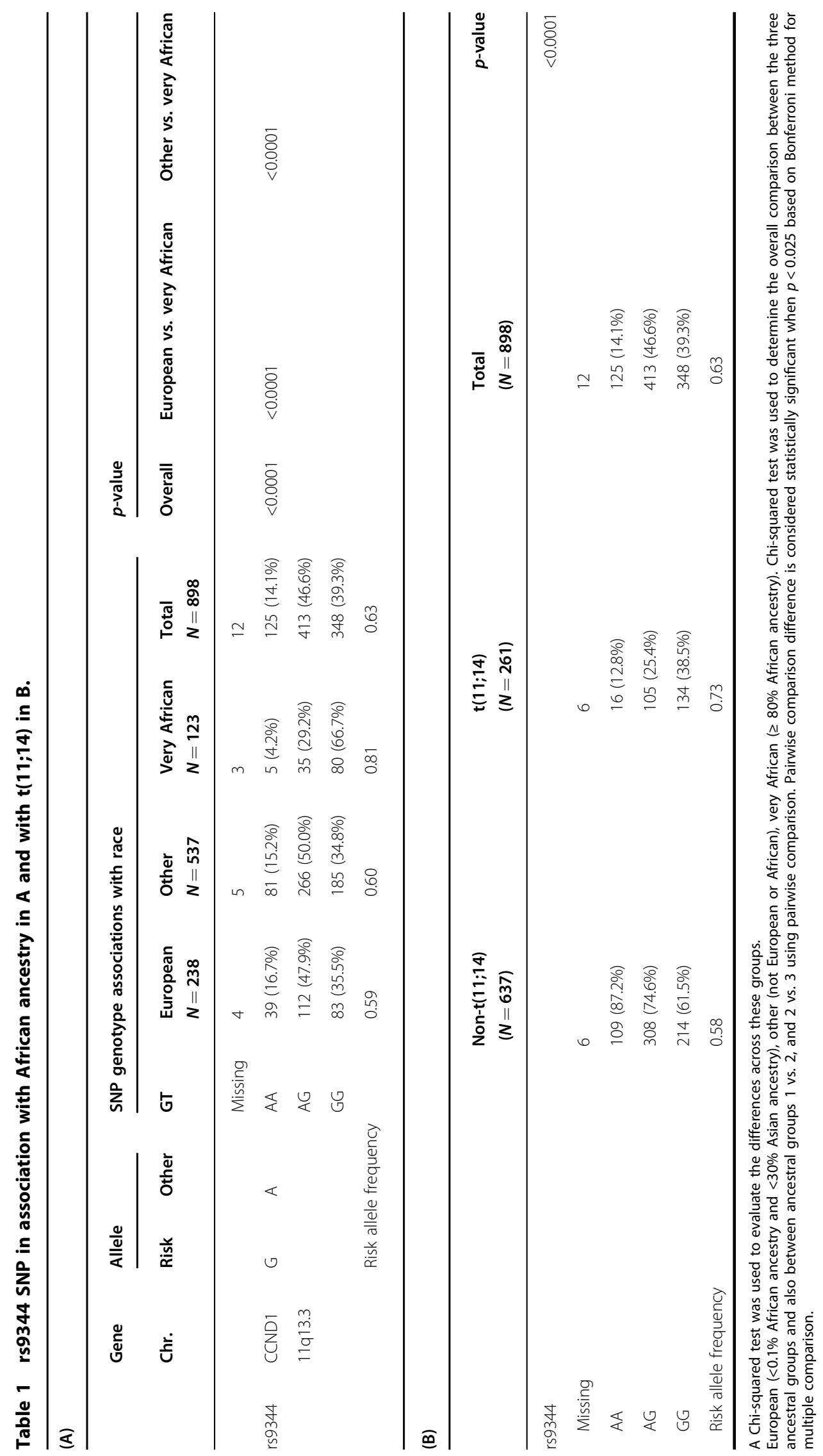


Table 2 Multivariate model predicting $t(11 ; 14)$.

\begin{tabular}{llll}
\hline Variable & Class & OR 95 Cl & $\boldsymbol{p}$-value \\
\hline Age & & $1.01(0.99,1.02)$ & 0.338 \\
Gender & Male & $1.57(1.15,2.14)$ & 0.004 \\
rs9344 & AG genotype & $2.27(1.28,4.04)$ & 0.005 \\
& GG genotype & $4.02(2.26,7.17)$ & $<0.001$ \\
Race & Very African & $1.17(0.69,1.97)$ & 0.564 \\
& Other & $1.04(0.72,1.49)$ & 0.845 \\
\hline
\end{tabular}

Variables included age, gender, race, and SNP rs9344

other (not European or African), very African ( $\geq 80 \%$ African ancestry). A logistic regression model was used to evaluate the differences.

plasma cell clone along with uniformly collected FISH, genotyping, and ancestry data. Utilization of the immunoglobulin kappa and lambda light chain counterstain during FISH analysis provide specificity allowing for the scoring of only light chain expressing plasma cells clones. Inclusion of only cytogenetically abnormal clones with recurrent $\mathrm{MM}$ abnormalities provides addition specificity at the risk of excluding clones with normal or nonrecurring cytogenetic abnormalities that our plasma cell FISH panels do not detect. Utilization of this population of patients with abnormal FISH studies, we have identified the association of the G-risk allele of CCND1 c.870G > A polymorphism (rs9344) with African ancestry and with $t(11 ; 14)$, suggesting that it may play a role in the development of $t(11 ; 14)$ plasma cell disorders. Future studies are needed to further understand the mechanism behind this association and also evaluate whether there exists differences in outcome and/or response to therapy between AAs and EAs with $\mathrm{t}(11 ; 14) \mathrm{MM}$.

\section{Acknowledgements}

Research reported in this publication was supported by the National Cancer Institute of the National Institutes of Health under Award Number P50CA186781 from the Mayo Clinic Multiple Myeloma Specialized Program of Research Excellence. The content is solely the responsibility of the authors and does not necessarily represent the official views of the National Institutes of Health. We also acknowledge support by the Mayo Clinic Department of Laboratory Medicine and Pathology, Center for Individualized Medicine and support of the Marion Schwartz Career Development Award in Multiple Myeloma. We thank Neeraj Sharma, Ph.D. for review of this manuscript.

\section{Author details}

'Division of Laboratory Genetics, Department of Laboratory Medicine and Pathology, Mayo Clinic, Rochester, MN, USA. 2Division of Biomedical Statistics and Informatics, Department of Health Sciences Research, Mayo Clinic, Jacksonville, FL, USA. ${ }^{3}$ Division of Biomedical Statistics and Informatics, Department of Health Sciences Research, Mayo Clinic, Rochester, MN, USA. ${ }^{4}$ Integrated Cancer Genomics, Translational Genomics Research Institute (TGen), Phoenix, AZ, USA. ${ }^{5}$ Department of Biology, Lund University, Lund, Sweden. ' $D N A$ Diagnostics Center, Fairfield, $\mathrm{OH}$, USA. 'Division of Hematology, Department of Internal Medicine, Mayo Clinic, Scottsdale, AZ, USA. ${ }^{8}$ Division of Hematology, Department of Internal Medicine, Mayo Clinic, Rochester, MN, USA
Conflict of interest

A.D. has received support from Celgene, Takeda, Prothena, Jannsen, Pfizer, Alnylam, GSK. E.E. is a consultant to the DDC and Michael Baird is the CSO of DDC.

\section{Publisher's note}

Springer Nature remains neutral with regard to jurisdictional claims in published maps and institutional affiliations.

Supplementary Information accompanies this paper at (https://doi.org/ 10.1038/s41408-020-0294-5).

Received: 23 August 2019 Revised: 7 February 2020 Accepted: 17 February 2020

Published online: 16 March 2020

\section{References}

1. Kumar, S. K. \& Rajkumar, S. V. The multiple myelomas - current concepts in cytogenetic classification and therapy. Nat. Rev. Clin. Oncol. 15, 409-421 (2018).

2. Landgren, O. et al. Monoclonal gammopathy of undetermined significance (MGUS) consistently precedes multiple myeloma: a prospective study. Blood. 113, 5412-5417 (2009).

3. Waxman, A. J. et al. Racial disparities in incidence and outcome in multiple myeloma: a population-based study. Blood. 116, 5501-5506 (2010).

4. Landgren, O. et al. Risk of monoclonal gammopathy of undetermined significance (MGUS) and subsequent multiple myeloma among African American and white veterans in the United States. Blood. 107, 904-906 (2006).

5. Landgren, O. et al. Prevalence of monoclonal gammopathy of undetermined significance among men in Ghana. Mayo Clin. Proc. 82, 1468-1473 (2007).

6. Landgren, O. et al. Racial disparities in the prevalence of monoclonal gammopathies: a population-based study of 12,482 persons from the National Health and Nutritional Examination Survey. Leukemia. 28, 1537-1542 (2014).

7. VanValkenburg, M. E. et al. Family history of hematologic malignancies and risk of multiple myeloma: differences by race and clinical features. Cancer Causes Control. 27, 81-91 (2016).

8. Ailawadhi, S. et al. Disease and outcome disparities in multiple myeloma: exploring the role of race/ethnicity in the Cooperative Group clinical trials. Blood Cancer J. 8, 67 (2018).

9. Manojlovic, Z. et al. Comprehensive molecular profiling of 718 Multiple Myelomas reveals significant differences in mutation frequencies between African and European descent cases. PLoS Genet. 13, e1007087 (2017).

10. Fillmore, N. R. et al. With equal access, African American patients have superior survival compared to white patients with multiple myeloma: a VA study. Blood. 133, 2615-2618 (2019).

11. Baughn, L. B. et al. Differences in genomic abnormalities among African individuals with monoclonal gammopathies using calculated ancestry. Blood Cancer J. 8, 96 (2018).

12. Kazandjian, D. et al. Molecular underpinnings of clinical disparity patterns in African American vs. Caucasian American multiple myeloma patients. Blood Cancer J. 9, 15 (2019).

13. Weinhold, N. et al. The CCND1 c.870G > A polymorphism is a risk factor for $t$ (11;14)(q13;q32) multiple myeloma. Nat. Genet. 45, 522-525 (2013).

14. Knudsen, K. E., Diehl, J. A., Haiman, C. A. \& Knudsen, E. S. Cyclin D1: polymorphism, aberrant splicing and cancer risk. Oncogene. 25, 1620-1628 (2006).

15. Bergsagel, P. L. \& Kuehl, W. M. Critical roles for immunoglobulin translocations and cyclin D dysregulation in multiple myeloma. Immunol. Rev. 194, 96-104 (2003).

16. Chesi, M. et al. Dysregulation of cyclin D1 by translocation into an lgH gamma switch region in two multiple myeloma cell lines. Blood. 88, 674-681 (1996).

17. Lu, F. et al. An alternatively spliced cyclin D1 isoform, cyclin D1b, is a nuclear oncogene. Cancer Res. 63, 7056-7061 (2003). 